\title{
Efficacy, safety and patient's quality of life of long-term treatment with levodopa-carbidopa intestinal gel in advanced Parkinson's disease in Romania: Results from GLORIA observational study
}

\author{
Jozsef Szasz' ${ }^{1}$ Mihaela Simu², Lacramioara Perju-Dumbrava ${ }^{3}$, Angelo Antonini ${ }^{4}$, \\ Lars Bergmann ${ }^{5}$, Diana Popescu ${ }^{6}$, Ovidiu Alexandru Bajenaru ${ }^{7}$ \\ ${ }^{1}$ Department of Neurology, Emergency Clinical County Hospital Mures, \\ "George Emil Palade" University of Medicine, Pharmacy, Science and Technology, \\ Targu-Mures, Romania \\ 2Department of Neurology, Clinical Emergency County Hospital, \\ "Victor Babes" University of Medicine and Pharmacy, Timisoara, Romania \\ 3"Iuliu Hatieganu" University of Medicine and Pharmacy, Cluj-Napoca, Romania \\ ${ }^{4}$ Department of Neuroscience, Padua University, Padua, Italy \\ ${ }^{5}$ AbbVie Inc., North Chicago, USA \\ ${ }^{6}$ AbbVie SLR, Bucharest, Romania \\ ${ }^{7}$ Department of Neurology, University Emergency Hospital, \\ "Carol Davila" University of Medicine and Pharmacy, Bucharest, Romania
}

\begin{abstract}
Objectives. The aim of the Global Long-Term Registry on Efficacy and Safety of DUODOPA in Patients with Advanced Parkinson's Disease in Routine Care (GLORIA) was to document 24-month efficacy, safety and the effect on patient's quality of life (QoL) of long-term treatment with levodopa-carbidopa intestinal gel (LCIG) in advanced Parkinson's disease (PD) in routine clinical care. Here we present the results for the patients enrolled in Romania in the GLORIA registry.

Material and methods. GLORIA registry is a multicenter, international, observational registry conducted between June 2010 and June 2015. In Romania, 39 patients with an indication for LCIG therapy were enrolled and followed for up to 24 months.

Outcomes. During the study period LCIG led to significant improvements in "Off" time, "On" time with dyskinesia, activities of daily living (ADLs), motor examination, non-motor symptoms, and QoL which maintained up to the end of follow-up. At 24 months, "Off" time had a mean reduction of $-5.2 \pm 3.1$ hours/day vs. baseline $(p<0.0001)$ and "On" time with dyskinesia had a mean reduction of $-3.5 \pm 3.3$ hours/day vs. baseline $(p<0.0001)$. Both ADLs and motor examination "On" scores showed a maximum improvement at 6 months and 12 months $(p<0.0001)$ and remained significantly lower vs. baseline at 24 months (ADLs -6.6 $\pm 8.7, p=0.0023$; motor examination $-6.4 \pm 7.3, p=0.0007$ ). Non-motor symptoms scale (NMSS) total score had a mean reduction at 24 months of -18.29 as compared to baseline $(p=0.0011)$. QoL, as assessed by PDQ-8 significantly improved at 6 months as compared to baseline $(p=$ $0.0141)$ and maintained its statistical significance until the 12 months evaluation $(p=0.0076)$. Adverse drug reactions possibly or probably related to the LCIG therapy were reported for $2.9 \%(1 / 35)$ of the patients during the temporary nasojejunal tube therapy and for $60.0 \%(21 / 35)$ of the patients during permanent tube phase.

Conclusions. LCIG treatment showed significant and clinically relevant long-term improvements in motor symptoms and QoL in advanced PD patients. The safety/tolerability data confirmed the established safety profile.
\end{abstract}

Keywords: Parkinson's disease, levodopa-carbidopa intestinal gel, efficacy, safety, quality of life 


\section{INTRODUCTION}

Parkinson's disease (PD) is a chronic, progressive, neurodegenerative disorder with an estimated prevalence in European populations of 1 to 2 per 1,000 persons (1). For Romania, available data indicate that over 70,000 persons are currently treated for PD (2). It is estimated that more than $1 \%$ of the population over age 65 are afflicted by PD (3) and its incidence and prevalence increase with age (4). Neuropathologically, PD is characterized by degeneration of dopamine-producing cells in the substantia nigra and manifests with slowness of voluntary movements, tremor and non-motor symptoms $(5,6)$.

Levodopa, a dopamine precursor, is the most efficacious therapy for PD, although its long-term use is associated with the development of motor complications (7). Furthermore, in advanced PD response fluctuations with wearing-off of levodopa effects and dyskinesia have been observed (8). They have been associated with unpredictable gastric emptying rates which contribute significantly to the large variations in levodopa plasma concentration profile seen in individual patients (9). To address this, treatment strategies that include infusions of various levodopa formulations have been developed and investigated in the past 30 years in an attempt to provide more continuous dopaminergic stimulation (10-12).

Levodopa-carbidopa intestinal gel (LCIG) was first introduced in Europe in 2004, and since then it has been approved in more than 40 countries worldwide for the treatment of levodopa-responsive advanced PD patients with severe motor fluctuations and hyper-/dyskinesia (8). This therapeutic approach is based on the continuous administration of levodopa/carbidopa into the small intestine. In contrast to oral therapy, gastric emptying is by-passed, and relatively steady plasma levels of LD are achieved with a resulting decrease in motor fluctuations and dyskinesia $(13,14)$.

Global Long-Term Registry on Efficacy and Safety of DUODOPA in Patients with Advanced Parkinson's Disease in Routine Care (GLORIA) was designed with the aim to document clinical efficacy and safety, the effect on patient's quality of life (QoL), and health economic outcomes of long term treatment with LCIG in patients with advanced PD in routine care (15). Here we present the results for the patients enrolled in Romania in the GLORIA registry.

\section{MATERIALS AND METHODS}

\section{Study design and population}

GLORIA registry was a multicenter, international, observational registry conducted between June 2010 and June 2015 in 75 movement disorders centers specialized for treatment of patients with advanced PD from 18 countries $(15,16)$. Of these centers, 7 participating centers were located in Romania.

Patients were eligible for inclusion in the GLORIA registry if they had an indication for LCIG therapy or had started this therapy within 12 months prior to study inclusion. The indication for LCIG therapy was advanced levodopa-responsive PD with severe motor fluctuations and hyper-/dyskinesia and for whom the existing therapies did not provide satisfactory relief of symptoms. Eligible patients were prescribed LCIG according to the summary of product characteristics and local standard of care and were followed for 24 months after the enrollment. LCIG therapy initiation and dose titration was performed via a temporary nasojejunal (NJ) tube and the administration continued through a permanent tube (PEG-J). For drug-naive patients, data was collected prospectively from the study enrollment up to 24 months, while for those with prior LCIG therapy, data was collected retrospectively for 12 months up to enrollment and for 24 months following the enrollment. Detailed study design was presented in the manuscript describing the main results of the GLORIA registry (15). Concomitant medications were permitted following the indication of the treating physician.

All patients provided written informed consent before any study-related procedures. The study was conducted in accordance with Good Clinical Practices guidelines, the Declaration of Helsinki, and local laws and regulations. For Romania, all study-related documents were approved by the National Ethics Committee for Medicine and Medical Devices.

\section{Efficacy assessment}

Efficacy outcomes included the changes in the activities of daily living, therapy complications, motor and non-motor symptoms and were collected at base- 
line (before therapy initiation with the temporary $\mathrm{NJ}$ tube), Day 1 (start of LCIG therapy and discharge following PEG-J placement) and every 6 months following the LCIG therapy initiation. The Unified Parkinson's Disease Rating Scale (UPDRS) (17) was used to evaluate changes in the activities of daily living (UPDRS II score, “On” state evaluation), complications of therapy (modified UPDRS IV items 32 and 39) and motor symptoms (UPDRS III score, "On” state evaluation). Non-motor symptoms were assessed using the NMS Scale (NMSS) (18).

\section{Quality of life assessment}

QoL information was collected at baseline (before therapy initiation with temporary NJ tube), Day 1 (start of LCIG therapy and discharge following PEG-J placement) and every 6 months following the LCIG therapy initiation. Disease-specific 8-item Parkinson's Disease Questionnaire (PDQ-8) (19) and EuroQoL-5 Dimensions (EQ-5D) (20) descriptive score and visual analog scale (VAS) were used to assess the QoL in the enrolled patients.

\section{Safety assessment}

Adverse drug reactions (ADRs), severe ADRs and medical device related complaints were collected from baseline up to 28 days following the end of the study, during temporary NJ tube and PEG-J phases. Device-related events that were associated with an impairment of a patient's health were counted as ADRs. ADRs were defined as all adverse events that had at least a reasonable possibility of a causal relationship to study treatment. All ADRs were coded according to the Medical Dictionary for Regulatory Activities (MedDRA) and categorized according to their severity (mild, moderate and severe) and according to their causal relationship with LCIG (unlikely, possible and probable).

\section{Statistical analyses}

Efficacy and QoL analyses were performed in the full analysis set population which included all enrolled patients that received at least one dose of LCIG, had a baseline efficacy evaluation and at least one post-baseline efficacy and safety evaluation. Safety analyses were performed in the safety analysis population which included all enrolled patients that received at least one dose of LCIG and had at least one post-baseline safety evaluation. The analyses of demographic data, medical history, concomitant medication and vital signs were performed on the population that included all patients that consented to participate, irrespective of the status of the post-baseline evaluations.

All recorded clinical observations were analyzed using descriptive statistics: number, percentage, mean and standard deviation. The comparison of efficacy and QoL data during the follow-up to baseline was performed by paired t-tests.

\section{RESULTS}

\section{Patients characteristics}

Of the 375 patients in the GLORIA registry, 39 were enrolled in centers from Romania. Of these, 35 had at least one post-baseline safety evaluation and were included in the safety analysis population. 32 had at least one post-baseline efficacy and safety evaluation and were included in the full analysis population. Patient characteristics at baseline are provided in Table 1.

\section{TABLE 1. Baseline patient characteristics}

\begin{tabular}{|l|c|}
\hline Patient characteristic & Baseline value \\
\hline Age, years & $62.2 \pm 8.2$ \\
\hline Male gender, n (\%) & $22(56.0 \%)$ \\
\hline BMI, kg/m & $24.9 \pm 4.1$ \\
\hline PD duration, years & $11.5 \pm 5.3$ \\
\hline Modified Hoehn and Yahr Staging & \\
Stage “On" & $3.2 \pm 0.6$ \\
Stage "Off” & $4.3 \pm 0.8$ \\
\hline LCIG naive, n (\%) & $26(67.0 \%)$ \\
\hline Previous PD therapy, n (\%) & \\
Levodopa & $37(94.9 \%)$ \\
COMT inhibitors & $31(79.5 \%)$ \\
Dopamine agonist & $30(76.9 \%)$ \\
MAO inhibitors & $27(69.2 \%)$ \\
Amantadine & $18(46.2 \%)$ \\
\hline Reasons for starting LCIG therapy, n (\%) & \\
Disable off periods & $37(94.9 \%)$ \\
Uncontrolled dyskinesia & $28(71.8 \%)$ \\
\hline Modified UPDRS IV, hours/day & \\
Item 32 (time with dyskinesia) & $4.7 \pm 3.4$ \\
Item 39 ("Off” time) & $6.7 \pm 2.9$ \\
\hline UPDRS II score "On” (activities of daily living) & $20.1 \pm 8.3$ \\
\hline UPDRS III score "On" (motor examination) & $20.2 \pm 6.2$ \\
\hline NMSS total score & $58.0 \pm 33.3$ \\
\hline
\end{tabular}

Results are displayed as mean \pm standard deviation, if not otherwise specified.

$B M I=$ body mass index; $C O M T=$ catechol-O-methyl transferase; $L C I G$ = levodopa-carbidopa intestinal gel; $M A O=$ monoamine oxidase; $\mathrm{N} / \mathrm{n}(\%)=$ number (percentage) of patients; $P D=$ Parkinson disease; UPDRS = Unified Parkinson's Disease Rating Scale; NMSS = non-motor symptom scale 
The mean age was 62.2 years, ranging between 44.0 and 76.0 years, the majority were men $(56.0 \%)$ and LCIG naive (67.0\%). The mean duration of PD was 11.5 years, ranging between 3.3 and 27.5 years. None of the patients had impulse control disorder and only $1(2.6 \%)$ fulfilled dementia criteria. At baseline, oral levodopa was the most frequently prescribed antiparkinsonian medication, followed by catechol-O-methyl transferase and dopamine agonists. None of them used apomorphine in either form or underwent deep brain stimulation for PD. During the follow-up, mean levodopa equivalent daily doses increased as compared to baseline with values ranging between $2,109.4$ to $2,504.3 \mathrm{mg}$ ( $\mathrm{p}$ for difference from baseline $<0.0001$ for all time points) for those who used LCIG in combination with other antiparkinsonian drugs and with values ranging between 1,765.8 and 2,195.0 $\mathrm{mg}$ (p for difference from baseline $<0.0001$ for all time points) for those who used LCIG monotherapy.

\section{Efficacy}

As compared to baseline, patients showed a significant and sustained reduction in "Off" time and "On" time with dyskinesia as of discharge following PEG-J placement (Day 1) through month 24. The mean reduction at 24 months was $-5.2 \pm 3.1$ hours $(\mathrm{p}<0.0001)$ for "Off" time and $-3.5 \pm 3.3$ hours $(\mathrm{p}<0.0001)$ for "On" time with dyskinesia, respectively. Also, improvements in activities of daily living (UPDRS II) and motor examination (UPDRS III) "On" scores were observed at all study visits as compared to baseline. Both activities of daily living and motor examination "On" scores showed a maximum decrease at 6 months (ADLs $-9.1 \pm 7.4, \mathrm{p}<0.0001$; motor examination $-8.4 \pm 7.1$, $\mathrm{p}<0.0001$ ) and 12 months (ADLs -9.3 $\pm 8.4, \mathrm{p}$ $<0.0001$; motor examination $-8.5 \pm 7.3, \mathrm{p}<0.0001)$, respectively and remained statistically significant lower than the baseline at 24 months (ADLs $-6.6 \pm 8.7, p=0.0023$; motor examination $-6.4 \pm 7.3$, $\mathrm{p}=0.0007)$. Compared to baseline the NMSS scores were significantly reduced at all study visits $(p<0.0001)$. The mean scores for Domains 3 (mood/cognition) and 6 (gastrointestinal tract) of the NMSS decreased statistically significant during the follow-up as compared to baseline and remained statistically significant lower than baseline values up to the last study visit (mean difference from baseline to month $24:-4.3$; $p$ for difference from baseline $=0.0039$ for Domain 3 and $-6.4 ; p$ for difference from baseline $=0.0006$ for Domain 6). Scores for Domain 2 (sleep/fatigue) were significantly lower than baseline scores only at 18 months $(\mathrm{p}=0.0033)$ with no significant improvements thereafter.

\section{Quality of life}

QoL, as assessed by PDQ-8 improved statistically significant at 6 months as compared to baseline $(p=0.0141)$ and the improvement maintained its statistical significance until the 12 months evaluation ( $\mathrm{p}=0.0076)$, with no statistically significant improvements thereafter. The mean scores in 4 items of the PDQ-8 showed a significant decrease at 6 months as compared to baseline: "Difficulty getting around in public places", "Difficulty dressing yourself", "Had painful muscle cramps and pains" and "Felt embarrassed by having PD". The reduction was persistent at 18 months for " $\mathrm{Had}$ painful muscle cramps and pains" and at 24 months for "Difficulty getting around in public places". EQ-5D total scores significantly improved at Day 1 and 6 months $((p=0.0079$ and 0.0188 , respectively $)$ as compared to baseline, with no statistically significant improvements thereafter. Per EQ-5D individual items, significant improvements of usual activities persisted until 18 months following baseline, while significant improvements of mobility and self-care persisted 6 months after baseline. Pain and discomfort items showed no improvement after LCIG therapy (Table 2).

\section{Safety}

During the temporary NJ tube therapy was reported 1 ADR occurring in 1 patient $(2.9 \%)$. This ADR (pneumoperitoneum) was considered serious, procedure-related, recovered before the end of follow-up and did not led to discontinuation of LCIG therapy.

During the PEG-J phase, 62.9\% (22/35) of the patients experienced at least 1 ADR and ADRs considered as possibly or probably related to the LCIG therapy were reported for $60.0 \%(21 / 35)$ of the patients. Moreover, serious ADRs occurred in $14.3 \%$ $(5 / 35)$ of the patients during this phase and included loss of consciousness (1 patient), parkinsonism (1 patient), polyneuropathy (1 patient), psychomo- 
TABLE 2. Mean change from baseline in the quality of life as assessed by PDQ-8 and EQ-5D scores (full analysis set population)

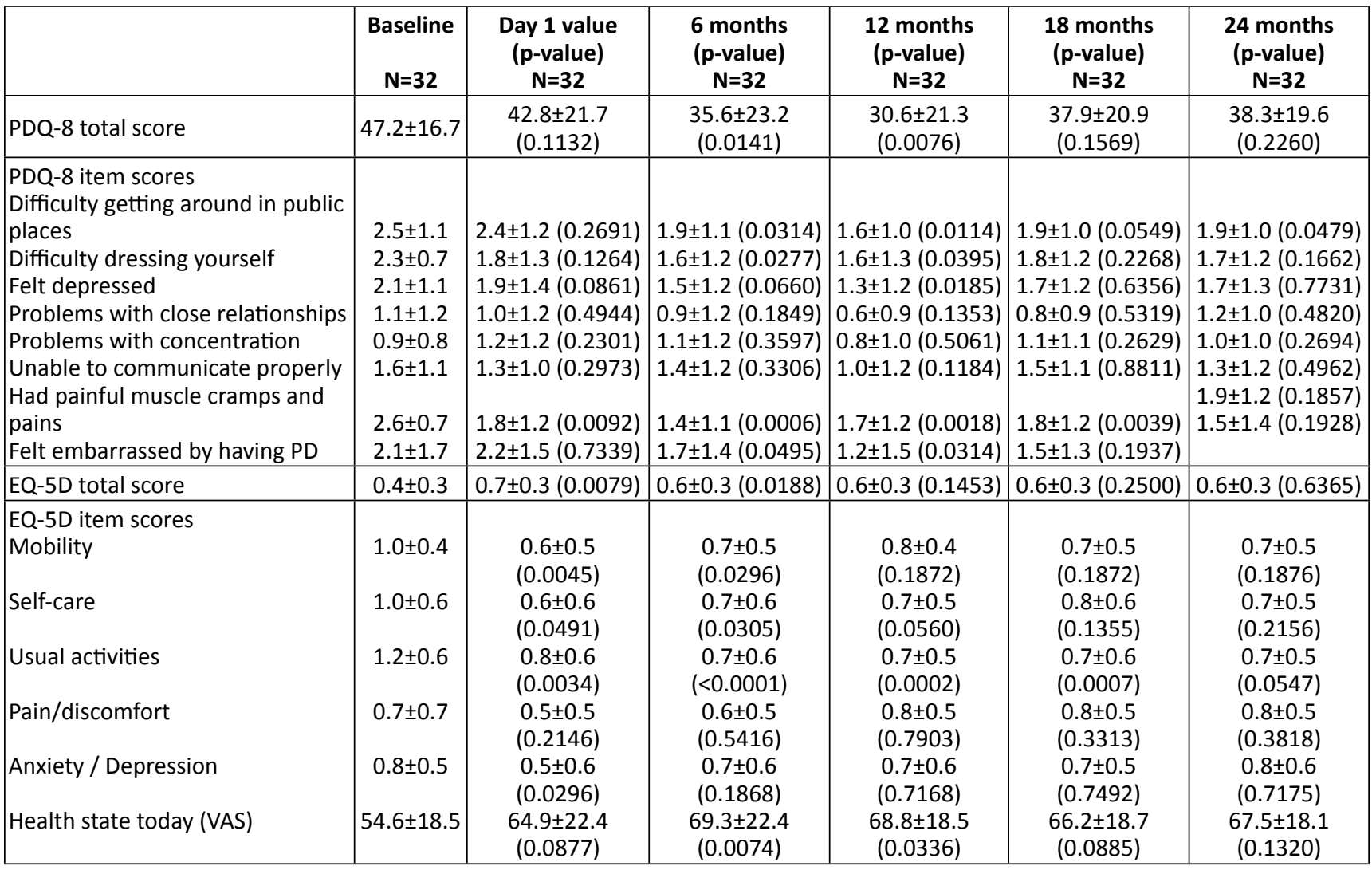

Note: Results are displayed as mean \pm standard deviation. All $p$-values were computed for the difference against baseline $N=$ number of patients; $P D Q-8=$ Parkinson's Disease Questionnaire 8-item; EQ-5D = EuroQoL- 5 Dimensions; VAS = visual analog scale

tor hyperactivity (1 patient) and pneumoperitoneum (1 patient, considered as device-related). The most frequently reported ADRs were decrease of body weight, polyneuropathy, peripheral sensorimotor neuropathy and increase of body weight (Table 3). No PEG-J procedure-related ADRs were reported during this phase. The rate of discontinuation of LCIG therapy in PEG-J phase due to ADRs was $2.9 \%$ ( 1 patient) and was due to the occurrence of neuropathy.

\section{DISCUSSIONS}

The analysis of data collected from Romanian patients enrolled in the GLORIA registry showed long-term improvements in motor complications as well as of non-motor symptoms and QoL in line with the whole study populations.

Over the entire 24-month LCIG treatment period there were stable and clinically relevant reductions in daily "Off" time and "On" time with dyskinesias, as assessed by modified UPDRS IV items 39 and 32 . The maximal reduction of 6.0 hours in daily "Off" time was observed at 6 months (p < 0.0001 ) and the maximal mean reduction of 3.9 hours in daily "On" time with dyskinesias was observed at 12 months $(\mathrm{p}<0.0001)$. These statistically significant reductions of the "Off" time and time with dyskinesia and long-term persistence of these improvements are comparable with previous results reported in interventional and observational clinical trials $(10,11,21-23)$ which showed that LCIG is an effective therapeutic option when aiming for long-term improvement of motor symptoms $(11,21,22)$. This study also confirms previous observations from a retrospective study in 113 patients with advanced PD from Romania, which showed a sustained reduction of motor symptoms after LCIG therapy initiation (24). It is important to note that the decrease in dyskinesia initially observed at discharge following PEG-J placement occurred despite significant increases in daily levodopa exposure after switching to LCIG infusion. This is consistent with previous clinical and preclinical data which showed that continuous dopaminergic stimulation is a key factor in the management of 
A

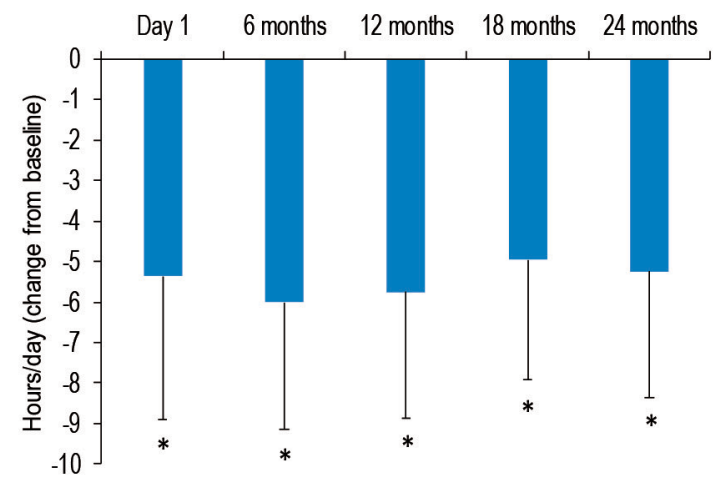

C

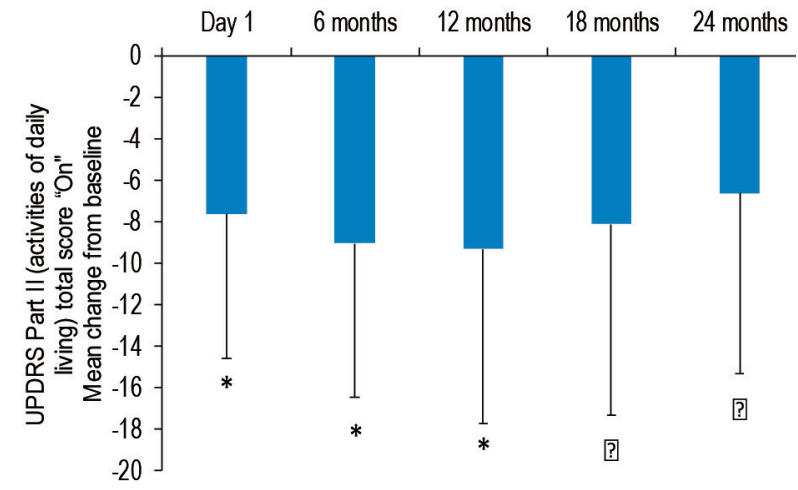

$\mathrm{E}$

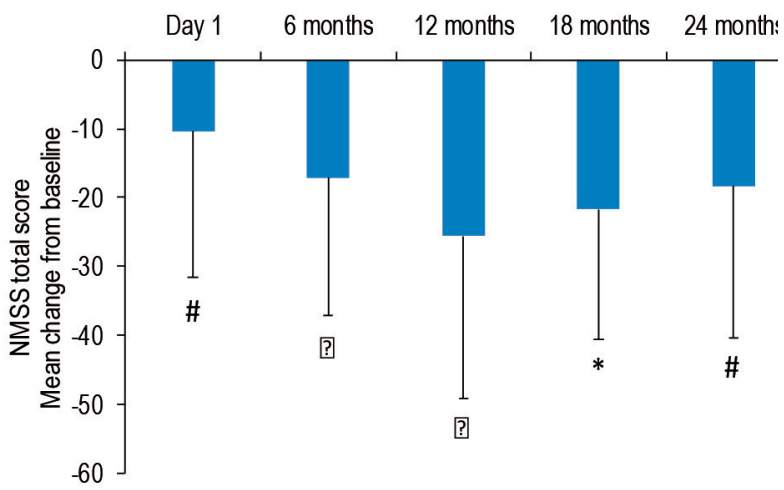

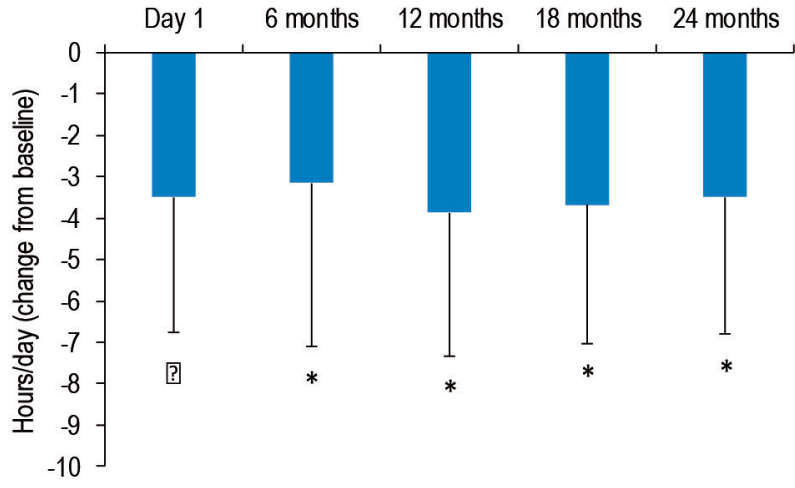

)

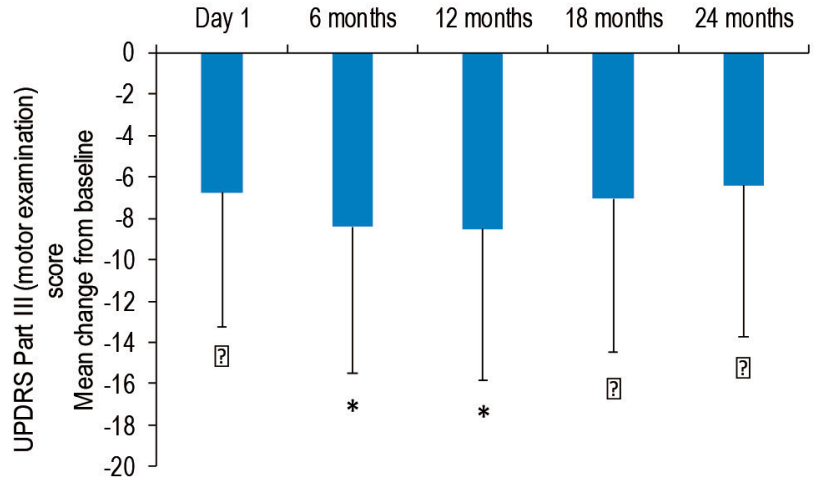

FIGURE 1. Changes from baseline in the "Off" time and "On" time with dyskinesia (panels A and B), activities of daily living (panel C), motor examination (panel $D$ ) and non-motor symptoms scores (panel E; full analysis set population)

${ }^{*} p<0.0001 ; \square p<0.001 ; \# p<0.05$

UPDRS = Unified Parkinson's Disease Rating Scale; NMSS = non-motor symptom scale

TABLE 3. Incidence of adverse drug reactions during the study (safety population)

\begin{tabular}{|c|c|c|}
\hline Type & $\begin{array}{l}\text { Temporary nasojejunal tube phase } \\
\qquad \mathrm{N}=35\end{array}$ & $\begin{array}{l}\text { Permanent tube treatment phase } \\
\qquad \mathrm{N}=35\end{array}$ \\
\hline Patients with at least 1 ADR, $n(\%)$ & $1(2.9 \%)$ & $22(62.9 \%)$ \\
\hline $\begin{array}{l}\text { Patients with at least } 1 \text { ADR possibly or } \\
\text { probably related to } L C I G, n(\%)\end{array}$ & $1(2.9 \%)$ & $21(60.0 \%)$ \\
\hline Serious ADR, $\mathrm{n}(\%)$ & $1(2.9 \%)$ & $5(14.3 \%)$ \\
\hline $\begin{array}{l}\text { ADRs reported in } \geq 3 \% \text { of the patients } \\
\text { Weight decreased } \\
\text { Polyneuropathy } \\
\text { Peripheral sensorimotor neuropathy } \\
\text { Weight increased }\end{array}$ & $\begin{array}{l}0 \\
0 \\
0 \\
0\end{array}$ & $\begin{array}{c}8(22.9 \%) \\
5(14.3 \%) \\
2(5.7 \%) \\
2(5.7 \%)\end{array}$ \\
\hline
\end{tabular}

$\mathrm{N} / \mathrm{n}(\%)=$ number (percentage) of patients; $A D R=$ adverse drug reaction; $L C I G$ = levodopa-carbidopa intestinal gel 
motor fluctuations and pre-existing dyskinesia $(10,25,26)$.

Also, the overall motor performance improved during the follow-up, as shown by 6.8 points decrease of UPDRS III score at the start of LCIG with a persistent reduction during the 2-year treatment period to end of the follow-up ( $p=0.0007)$. Furthermore, activities of daily living improved as shown by 6.6 points reduction of the UPDRS II score at the end of the follow-up $(p=0.0023)$. The improvements in the motor performance and activities of daily living were comparable to those reported by Fernandez et al. (11) in 354 patients with PD followed for 12 months. In this study the UPDRS II and III scores improved significantly at 4 weeks following the LCIG initiation and remained statistically significant lower as compared to baseline up to the end of the follow-up (11).

Consistent with GLORIA main results, in the sub-group analyzed here the NMSS overall scores showed sustained improvement during the follow-up. The domains that showed persistent improvements during the study were $\mathrm{mood} /$ cognition and gastrointestinal tract. Although, non-motor symptoms are considered as important determinants of the QoL in all stages of PD (27), they frequently go unrecognized by healthcare providers and thus remain untreated (25). The decrease of non-motor burden observed in our analysis was similar to the improvements reported in other longterm open-label LCIG therapy studies over 12- and 48-months follow-up duration $(28,29)$. Also, they are consistent with previous concept of improvement of non-motor symptoms with optimized dopaminergic therapy (30).

The beneficial effects observed on motor and non-motor outcomes were reflected by an improvement in QoL. These improvements were shown by the persistent reduction of the PDQ-8 total score up to 12 months post LCIG therapy initiation $(p=0.0076)$, persistent improvements to 18 and 24 months for "Had painful muscle cramps and pains" and "Difficulty getting around in public places", respectively, as well as a significant amelioration of the EQ-5D usual activities item score for 18 months following the baseline. Similar improvements in the QoL were previously reported following LCIG therapy $(10,11,23)$. A phase 3 study in which PD patients were randomized to either immediate-re- lease oral levodopa-carbidopa or LCIG showed statistically significant improvements of PDQ-39 with LCIG that maintained up to the end of 12 weeks follow-up period (10). More recently, in a 54-week study enrolling 354 patients, Fernandez et al. (11) reported PDQ-39 score improvement of 6.9 points ( $\mathrm{N}=273$ completed patients) and EQ-5D score improvement of $0.06(\mathrm{~N}=272$ completed patients) from prior to LCIG infusion to the study end. In our analysis we observed a diminished improvement over time in PDQ-8 scores and these may reflect PD progression, comorbidities, treatment-related ADRs, or development of treatment-resistant symptoms (16).

Apart from confirming the efficacy, this analysis also confirmed the established overall safety profile of LCIG, throughout the treatment period of 24 months. No new pattern or trend was observed in ADRs in patients receiving LCIG therapy and ADRs observed were similar to those reported in previous LCIG studies (31-33). The number of procedure and device-related ADRs was low in the Romanian sample. In this study, were reported only 1 procedure-related ADR (occurring during NJ tube therapy) and 1 device-related ADR (occurring during PEG-J phase) both considered as serious. The comparison of occurrence of these ADRs with other prospective studies is limited by the design of GLORIA registry that allowed the retrospective collection of data (15). However, the occurrence of procedure-related ADRs was in line with complications of the PEG-J placement procedure previously reported in the literature (33). The complications, although rare, may be life threatening and include pneumoperitoneum, peritonitis and intestinal perforation (33). The most frequently reported ADRs in the Romanian sample of the GLORIA registry were decrease of body weight $(22.9 \%)$ and polyneuropathy (14.3\%). Also, in only 1 patient the ADR (polyneuropathy) led to LCIG therapy discontinuation. Weight loss and polyneuropathy have been frequently reported in other clinical trials with LCIG in PD (31-33). In a retrospective study of 900 patients and a prospective long-term follow-up of 252 PD patients treated with LCIG, weight loss rates observed were around $14.0 \%$ while polyneuropathy rates were around 3.0\% (31,32). Similarly, in an integrated safety analysis of prospective clinical trials which included 412 patients with a medi- 
an LCIG exposure of 911 days weight loss was reported for $14.0 \%$ of the treated patients and polyneuropathy for $5.5 \%$ of the treated patients (33). Polyneuropathy has been considered as a possible complication of LCIG infusion, however, the etiology remains uncertain with hyperhomocysteinemia and low levels of vitamin B6, vitamin B12, folic acid among the presumed mechanisms involved in its development (34). It is important to note that this study did neither include formalized assessment of peripheral nerve conduction velocity to confirm diagnoses nor were laboratory assessments conducted for vitamin B12, folic acid or homocysteine evaluation.

This registry bears some design-related limitations, in particular, its non-controlled, observational nature. Nonetheless this design allowed the collection of clinical data derived from a population of advanced PD patients treated in clinical routine care allowing insights in the real-world use and outcomes of LCIG. Allowing partially retrospective (up to 12 months) and prospective data collection may represent another potential limitation. Not all scales and questionnaires were used by all investigational sites prior to participation in GLORIA resulting in missing data during the retrospectively documented treatment period.

\section{CONCLUSIONS}

In this long-term registry, LCIG treatment showed significant and clinically relevant improvements in motor and non-motor symptoms and QoL in advanced PD patients. LCIG was in general well tolerated, with an overall favorable benefit/risk ratio consistent with its previously established safety profile.

Funding: Global study was sponsored by AbbVie Inc. (North Chicago, IL, USA). Romanian data sub-analysis reported here was sponsored by AbbVie SLR (Bucharest, Romania).

\section{Conflict of interest}

Prof. Szasz was a study investigator and declares speaker fees from AbbVie, Novartis, UCB,
Boehringer-Ingelheim, GSK, Ever, Lundbeck, Teva and Pfizer

Prof. Simu has received honoraria for lectures in symposia and consultancy from Teva, AbbVie, Merck, Servier Pharma, AOP Orphan, BoehringerIngelheim, Sanofi, Krka, and UCB Pharma.

Prof. Perju-Dumbrava has received honoraria as investigator in clinical studies from Koehler eClinical Gmbh, PSI CRO AG, as well as honoraria for lectures from Sanofi Aventis, Solpharm, AbbVie, Boehringer-Ingelheim, Bristol Myers, Novartis Pharma, Berlin Chemie, Servier Pharma, Stada M\&D, UCB Pharma and as member of the advisory board for AbbVie.

Prof. Antonini was a study investigator and declares speaker and consultancy fees from UCB, Boehringer Ingelheim, AbbVie, Zambon, Bial, Ever Pharma, GE, Neuroderm, Therevance, Biogen; he receives research support from Chiesi Pharmaceuticals, Lundbeck, Horizon 2020 - PD_Pal Grant 825785, Ministry of Education University and Research (MIUR) Grant ARS01_01081. He serves as consultant for Boehringer-Ingelheim for legal cases on pathological gambling".

Dr. Bergmann is an employee of AbbVie.

Dr. Popescu is an employee of AbbVie.

Prof. Bajenaru received speaker and consulting fees from AbbVie SLR, and speaker fees from Lundbeck, UCB Pharma and Boehringer-Ingelheim.

\section{AbbVie Disclosures}

GLORIA registry was sponsored by AbbVie Inc. (North Chicago, IL, USA). AbbVie sponsored the study; contributed to the design; participated in collection, analysis, and interpretation of data; and in writing, reviewing, and approval of the final version.

\section{Acknowledgements}

Medical writing support for this work was provided by MedInteractiv Plus on behalf of AbbVie SLR. 


\section{REFERENCES}

1. von Campenhausen $S$, Bornschein B, Wick R et al. Prevalence and incidence of Parkinson's disease in Europe. Eur Neuropsychopharmacol. 2005;15:473-90.

2. Antiparkinson Association Romania. Available at: http://www. asociatia-antiparkinson.ro/Boala-Parkinson-HTML/Despre-boalaParkinson.html\#statistici-boala-Parkinson. Last accessed 29 Nov 2019.

3. Tysnes OB, Storstein A. Epidemiology of Parkinson's disease. J Neural Transm (Vienna). 2017;124:901-5.

4. Pringsheim T, Jette N, Frolkis A, Steeves TD. The prevalence of Parkinson's disease: A systematic review and meta-analysis. Mov Disord. 2014;29:1583-90.

5. Braak H, Del Tredici K, Rub U et al. Staging of brain pathology related to sporadic Parkinson's disease. Neurobiol Aging. 2003; 24:197-211.

6. Garcia-Ruiz PJ, Chaudhuri KR, Martinez-Martin P. Non-motor symptoms of Parkinson's disease A review... from the past. J Neurol Sci. 2014;338:30-3.

7. Sarkar S, Raymick J, Imam S. Neuroprotective and Therapeutic Strategies against Parkinson's Disease: Recent Perspectives. Int $J$ Mol Sci. 2016;17.

8. Wirdefeldt K, Odin P, Nyholm D. Levodopa-Carbidopa Intestinal Gel in Patients with Parkinson's Disease: A Systematic Review. CNS Drugs. 2016;30:381-404.

9. Contin M, Martinelli P. Pharmacokinetics of levodopa. J Neurol. 2010;257(Suppl 2):S253-61.

10. Olanow CW, Kieburtz K, Odin $P$ et al. Continuous intrajejunal infusion of levodopa-carbidopa intestinal gel for patients with advanced Parkinson's disease: a randomised, controlled, double-blind, double-dummy study. Lancet Neurol. 2014;13:141-9.

11. Fernandez HH, Standaert DG, Hauser RA et al. Levodopa-carbidopa intestinal gel in advanced Parkinson's disease: final 12-month, open-label results. Mov Disord. 2015;30:500-9.

12. Bredberg E, Tedroff J, Aquilonius SM Paalzow L. Pharmacokinetics and effects of levodopa in advanced Parkinson's disease. Eur J Clin Pharmacol. 1990;39:385-9.

13. Nyholm D, Askmark H, Gomes-Trolin $\mathrm{C}$ et al. Optimizing levodopa pharmacokinetics: intestinal infusion versus oral sustained-release tablets. Clin Neuropharmacol. 2003;26:156-63.

14. Nyholm D, Odin P, Johansson A et al. Pharmacokinetics of levodopa, carbidopa, and 3-O-methyldopa following 16-hour jejunal infusion of levodopa-carbidopa intestinal gel in advanced Parkinson's disease patients. AAPS J. 2013;15:316-23.

15. Antonini A, Poewe W, Chaudhuri KR et al. Levodopa-carbidopa intestinal gel in advanced Parkinson's: Final results of the GLORIA registry. Parkinsonism Relat Disord. 2017;45:13-20.

16. Antonini A, Robieson WZ, Bergmann L et al. Age/disease duration influence on activities of daily living and quality of life after levodopacarbidopa intestinal gel in Parkinson's disease. Neurodegener Dis Manag. 2018;8:161-70.

17. Goetz CG, Tilley BC, Shaftman SR et al. Movement Disorder Society-sponsored revision of the Unified Parkinson's Disease Rating Scale (MDS-UPDRS): scale presentation and clinimetric testing results. Mov Disord. 2008;23:2129-70.

18. Chaudhuri KR, Martinez-Martin P, Schapira AH et al. International multicenter pilot study of the first comprehensive self-completed non-motor symptoms questionnaire for Parkinson's disease: the NMSQuest study. Mov Disord. 2006;21:916-23.

19. Jenkinson C, Fitzpatrick R. Cross-cultural evaluation of the short form 8-item Parkinson's Disease Questionnaire (PDQ-8): results from America, Canada, Japan, Italy and Spain. Parkinsonism Relat Disord. 2007;13:22-8.

20. EuroQol G. EuroQol--a new facility for the measurement of health-related quality of life. Health Policy. 1990;16:199-208.

21. Antonini A, Fung VS, Boyd JT et al. Effect of levodopa-carbidopa intestinal gel on dyskinesia in advanced Parkinson's disease patients. Mov Disord. 2016;31:530-7.

22. Slevin JT, Fernandez HH, Zadikoff $\mathrm{C}$ et al. Long-term safety and maintenance of efficacy of levodopa-carbidopa intestinal gel: an open-label extension of the double-blind pivotal study in advanced Parkinson's disease patients. J Parkinsons Dis. 2015;5:165-74.

23. Antonini A, Odin P, Opiano L et al. Effect and safety of duodenal levodopa infusion in advanced Parkinson's disease: a retrospective multicenter outcome assessment in patient routine care. J Neural Transm (Vienna). 2013;120:1553-8.

24. Băjenaru O, Ene A, Popescu BO et al. The effect of levodopa-carbidopa intestinal gel infusion long-term therapy on motor complications in advanced Parkinson's disease: a multicenter Romanian experience. J Neural Transm (Vienna). 2016;123:407-14.

25. Antonini A, Chaudhuri KR, Martinez-Martin P, Odin P. Oral and infusion levodopa-based strategies for managing motor complications in patients with Parkinson's disease. CNS Drugs. 2010; 24:119-29.

26. Timpka J, Fox T, Fox K et al. Improvement of dyskinesias with L-dopa infusion in advanced Parkinson's disease. Acta Neurol Scand. 2016;133:451-8.

27. Santos-García D, de la Fuente-Fernández R. Impact of non-motor symptoms on health-related and perceived quality of life in Parkinson's disease. J Neurol Sci. 2013;332:136-40.

28. Juhász A, Aschermann Z, Ács $P$ et al. Levodopa/carbidopa intestinal gel can improve both motor and non-motor experiences of daily living in Parkinson's disease: an open-label study. Parkinsonism Relat Disord. 2017:37:79-86.

29. Bohlega S, Abou Al-Shaar H, Alkhairallah T et al. Levodopa-carbidopa intestinal gel infusion therapy in advanced Parkinson's disease: single Middle Eastern center experience. Eur Neurol. 2015; 74:227-36.

30. Antonini A, Albin RL. Dopaminergic treatment and non-motor features of Parkinson disease: the horse lives. Neurology. 2013;80:784-5.

31. Fernandez HH, Boyd JT, Fung VSC et al. Long-term safety and efficacy of levodopa-carbidopa intestinal gel in advanced Parkinson's disease. Mov Disord. 2018;33:928-36.

32. Sensi M, Cossu G, Mancini F et al. Which patients discontinue? Issues on Levodopa/carbidopa intestinal gel treatment: Italian multicentre survey of 905 patients with long-term follow-up. Parkinsonism Relat Disord. 2017;38:90-2.

33. Lang AE, Rodriguez RL, Boyd JT et al. Integrated safety of levodopa-carbidopa intestinal gel from prospective clinical trials. Mov Disord. 2016;31:538-46.

34. Merola A, Zibetti M, Rizzone MG et al. Prospective assessment of peripheral neuropathy in Duodopa-treated parkinsonian patients. Acta Neurol Scand. 2014;129:e1-5. 